General Mathematics Vol. 27, No. 2 (2019), 23-34

DOI:10.2478/gm-2019-0012

Sciendo

\title{
On Some Common Approximate Fixed Point Theorems
}

\author{
Kushal Roy, Mantu Saha
}

\begin{abstract}
In the present paper we establish some theorems on the existence of common approximate fixed points for a pair of generalized contractive type mappings with the property that the diameter of the set of common $\epsilon$-fixed points is tending to zero as $\epsilon$ tends to zero.
\end{abstract}

2010 Mathematics Subject Classification: 47H10, 54H25.

Key words and phrases: generalized contractive mapping, approximate fixed point, common approximate fixed point, common approximate fixed point property.

\section{Introduction}

The study of fixed point theory finds a great deal of importance due to its wide applicability in non-linear analysis. The conditions imposed in fixed point theorems are so strong that the existence of fixed point might not be guaranteed. So it is natural to study on weakening the fixed point property by approximate fixed points of mappings. A point $x$ of a mapping $f$ is an approximate fixed point of $f$, is to mean ' $f(x)$ is near to $x$ '. The study of approximate fixed points for mappings has equally importance to the study of fixed point of mapping. The idea of approximate fixed point was first studied by Tijs et. al. in [8], following which M. Berinde [1] proved some important approximate fixed point theorems over usual metric spaces. Later

\footnotetext{
${ }^{1}$ Received 8 June, 2018

Accepted for publication (in revised form) 6 November, 2019
} 
on many researchers have proved several approximate fixed point theorems using various contractive type mappings over different metric type spaces (See [4], [6]). In 2013, Dey and Saha [2] had proved some approximate fixed point theorems for Reich operator generalizing the theorems due to Berinde [1]. In the same year, Dey et. al. [3] proved some approximate coincidence point theorems for non-linear single valued mappings, following the work of W. S. Du [5] on approximate coincidence points of various multivalued mappings. The aim of this paper is to establish the existence of common approximate fixed points for a pair of generalized contractive mappings over a metric space without having completeness property.

\section{Basic ideas and Preliminaries}

In this section we recall the following basic preliminaries.

Definition 1 [1] Let $f: X \rightarrow X, \epsilon>0, x_{0} \in X$. Then $x_{0}$ is an $\epsilon$-fixed point (approximate fixed point) of $f$ if $d\left(f x_{0}, x_{0}\right)<\epsilon$.

Remark 1 [1] In this paper we will denote the set of all $\epsilon$-fixed points of $f$, for a given $\epsilon>0$, by $F_{\epsilon}(f)=\{x \in X: x$ is an $\epsilon$-fixed point of $f\}$.

Definition 2 [1] Let $f: X \rightarrow X$. Then $f$ has the approximate fixed point property (a.f.p.p.) if for all $\epsilon>0, F_{\epsilon}(f) \neq \emptyset$.

Remark 2 [1] The following result gives conditions under which the existence of fixed points for a given mapping is equivalent to that of approximate fixed points.

Proposition 1 [1] let $A$ be a closed subset of a metric space $(X, d)$ and $f: A \rightarrow X$ a compact map. Then $f$ has a fixed point if and only if it has the approximate fixed point property.

We also assume $\delta(A)$ as the diameter of a set $A \neq \emptyset$, i.e. $\delta(A)=\sup \{d(x, y)$ : $x, y \in A\}$.

Lemma 1 [1] Let $(X, d)$ be a metric space, $f: X \rightarrow X$ such that $f$ is asymptotically regular, i.e., $d\left(f^{n} x, f^{n+1} x\right) \rightarrow 0$ as $n \rightarrow \infty$, for all $x \in X$. Then $f$ has approximate fixed point property.

Lemma 2 [1] Let $(X, d)$ be a metric space, $f: X \rightarrow X$ an operator and $\epsilon>0$. We assume that

(i) $F_{\epsilon}(f) \neq \emptyset$;

(ii) $\forall \eta>0, \exists \phi(\eta)>0$ such that $d(x, y)-d(f x, f y) \leq \eta$ implies $d(x, y) \leq \phi(\eta)$, for all $x, y \in F_{\epsilon}(f)$. Then $\delta\left(F_{\epsilon}(f)\right) \leq \phi(2 \epsilon)$. 


\section{Main Results}

We first define the common approximate fixed point of a pair of mappings over a metric space $(X, d)$.

Definition 3 Let $(X, d)$ be a metric space, $\epsilon>0$ and $T, S: X \rightarrow X$ be two mappings. Then a point $x_{0} \in X$ is said to be a common approximate fixed point (common $\epsilon$-fixed point) of $T$ and $S$ if $d\left(T x_{0}, x_{0}\right)<\epsilon$ and also $d\left(S x_{0}, x_{0}\right)<\epsilon$.

Throughout this paper we denote the set of all common approximate fixed points (common $\epsilon$-fixed points) of $T$ and $S$ by $F_{\epsilon}(T, S)$. Clearly $F_{\epsilon}(T, S)=F_{\epsilon}(T) \cap F_{\epsilon}(S)$.

Definition 4 Two mappings $T, S$ in a metric space $(X, d)$ are said to have the common approximate fixed point property (c.a.f.p.p.) if for any $\epsilon>0, F_{\epsilon}(T, S) \neq \emptyset$.

Lemma 3 Let $(X, d)$ be a metric space and $T, S: X \rightarrow X$ be two mappings. Also let $d\left(x_{n}, x_{n+1}\right) \rightarrow 0$ as $n \rightarrow \infty$, where $\left\{x_{n}\right\}$ is given by

$$
x_{n}= \begin{cases}T x_{n-1}, & \text { when } n \text { is odd } \\ S x_{n-1}, & \text { when } n \text { is even }\end{cases}
$$

for some $x_{0} \in X$. Then for any $\epsilon>0, F_{\epsilon}(T) \neq \emptyset$ and $F_{\epsilon}(S) \neq \emptyset$ implies that both $T$ and $S$ have the approximate fixed point property.

Proof. Since $d\left(x_{n}, x_{n+1}\right) \rightarrow 0$ as $n \rightarrow \infty$ then for a given $\epsilon>0$ there exists $N \in \mathbb{N}$ such that $d\left(x_{n}, x_{n+1}\right)<\epsilon$ whenever $n \geq N$.

So there exists $m_{1}, m_{2} \in \mathbb{N}$ such that $d\left(x_{2 m_{1}}, x_{2 m_{1}+1}\right)<\epsilon$ and $d\left(x_{2 m_{2}-1}, x_{2 m_{2}}\right)<\epsilon$, implying that $d\left(x_{2 m_{1}}, T x_{2 m_{1}}\right)<\epsilon$ and $d\left(x_{2 m_{2}-1}, S x_{2 m_{2}-1}\right)<\epsilon$. Denoting $x_{2 m_{1}}$ by $y$ and $x_{2 m_{2}-1}$ by $z$ we get, $d(y, T y)<\epsilon$ and $d(z, S z)<\epsilon$. Thus $y \in F_{\epsilon}(T)$ and $z \in F_{\epsilon}(S)$. Hence $F_{\epsilon}(T) \neq \emptyset$ and $F_{\epsilon}(S) \neq \emptyset$ i.e. both $T, S$ have the a.f.p.p. .

Lemma 4 Let $(X, d)$ be a metric space and $T, S: X \rightarrow X$ be two mappings on $X$. Let us assume that the following conditions are satisfied.

(i) For any $\epsilon>0, F_{\epsilon}(T) \neq \emptyset$ and $F_{\epsilon}(S) \neq \emptyset$ that is both $T$ and $S$ have the approximate fixed point property;

(ii) For each $\epsilon>0$, there exists a mapping $\phi_{\epsilon}$ such that $\phi_{\epsilon}(\eta)>0$ for all $\eta>0$ and $d(x, y)-d(T x, S y) \leq \eta \Rightarrow d(x, y) \leq \phi_{\epsilon}(\eta)$ for all $x \in F_{\epsilon}(T)$ and $y \in F_{\epsilon}(S)$. Then $\delta\left(F_{\epsilon}(T, S)\right) \leq \phi_{\epsilon}(2 \epsilon)$, if $T$ and $S$ have the c.a.f.p.p. .

Proof. Let $\epsilon>0$ be given. Let $T$ and $S$ have the c.a.f.p.p. . Then for any $\epsilon>0$, $F_{\epsilon}(T) \neq \emptyset$ and $F_{\epsilon}(S) \neq \emptyset$. Let us choose $x, y \in F_{\epsilon}(T, S)$. Then $x \in F_{\epsilon}(T)$ and $y \in F_{\epsilon}(S)$, i.e. $d(x, T x)<\epsilon$ and $d(y, S y)<\epsilon$.

Now, $d(x, y) \leq d(x, T x)+d(T x, S y)+d(S y, y)$ implies that $d(x, y)-d(T x, S y) \leq$ $d(x, T x)+d(y, S y)<2 \epsilon$; and therefore by given condition (ii) we have $d(x, y) \leq$ $\phi_{\epsilon}(2 \epsilon)$. Since $x, y \in F_{\epsilon}(T, S)$ is arbitrary it follows that $\delta\left(F_{\epsilon}(T, S)\right) \leq \phi_{\epsilon}(2 \epsilon)$.

Next we prove some theorems using different contractive conditions for pair of mappings due to [7]. 
Theorem 1 Let $(X, d)$ be a metric space and $T, S: X \rightarrow X$ be two mappings such that $d(T x, S y) \leq \alpha[d(x, T x)+d(y, S y)] \forall x, y \in X, 0<\alpha<\frac{1}{2}$. Then for any $\epsilon>0$, $F_{\epsilon}(T) \neq \emptyset$ and also $F_{\epsilon}(S) \neq \emptyset$; that is both $T$ and $S$ have the approximate fixed point property.

Proof. Let $x_{0} \in X$. We construct the sequence $\left\{x_{n}\right\}$ on $X$ by

$$
x_{n}= \begin{cases}T x_{n-1}, & \text { when } n \text { is odd } \\ S x_{n-1}, & \text { when } n \text { is even }\end{cases}
$$

Then,

$$
\begin{aligned}
d\left(x_{1}, x_{2}\right)=d\left(T x_{0}, S x_{1}\right) & \leq \alpha\left[d\left(x_{0}, T x_{0}\right)+d\left(x_{1}, S x_{1}\right)\right] \\
& \left.=\alpha\left(x_{0}, x_{1}\right)+d\left(x_{1}, x_{2}\right)\right]
\end{aligned}
$$

Thus, $d\left(x_{1}, x_{2}\right) \leq \frac{\alpha}{1-\alpha} d\left(x_{0}, x_{1}\right)$. Also,

$$
\begin{aligned}
d\left(x_{2}, x_{3}\right)=d\left(S x_{1}, T x_{2}\right) & \leq \alpha\left[d\left(x_{2}, T x_{2}\right)+d\left(x_{1}, S x_{1}\right)\right] \\
& \left.=\alpha\left(x_{2}, x_{3}\right)+d\left(x_{1}, x_{2}\right)\right]
\end{aligned}
$$

Therefore, $d\left(x_{2}, x_{3}\right) \leq \frac{\alpha}{1-\alpha} d\left(x_{1}, x_{2}\right) \leq\left(\frac{\alpha}{1-\alpha}\right)^{2} d\left(x_{0}, x_{1}\right)$.

Proceeding similarly we get, $d\left(x_{n}, x_{n+1}\right) \leq\left(\frac{\alpha}{1-\alpha}\right)^{n} d\left(x_{0}, x_{1}\right) \forall n \in \mathbb{N}$. Then, $d\left(x_{n}, x_{n+1}\right)$ $\rightarrow 0$ as $n \rightarrow \infty$. Therefore by Lemma 3 we get both $T$ and $S$ have the approximate fixed point property.

Theorem 2 Let $(X, d)$ be a metric space and $T, S: X \rightarrow X$ be two self maps such that $d(T x, S y) \leq \alpha[d(x, S y)+d(y, T x)] \forall x, y \in X, 0<\alpha<\frac{1}{2}$. Then for any $\epsilon>0$, $F_{\epsilon}(T) \neq \emptyset$ and $F_{\epsilon}(S) \neq \emptyset$ implying that both $T$ and $S$ have the approximate fixed point property.

Proof. Let $x_{0} \in X$. We construct the sequence $\left\{x_{n}\right\}$ in $X$, defined by $x_{2 n}=S_{2 n-1}$ and $x_{2 n-1}=T x_{2 n-2}$ for all $n \geq 1$. Then,

$$
\begin{aligned}
d\left(x_{1}, x_{2}\right)=d\left(T x_{0}, S x_{1}\right) & \leq \alpha\left[d\left(x_{0}, S x_{1}\right)+d\left(x_{1}, T x_{0}\right)\right] \\
& =\alpha d\left(x_{0}, x_{2}\right) \\
& \leq \alpha\left[d\left(x_{0}, x_{1}\right)+d\left(x_{1}, x_{2}\right)\right]
\end{aligned}
$$

Thus, $d\left(x_{1}, x_{2}\right) \leq \frac{\alpha}{1-\alpha} d\left(x_{0}, x_{1}\right)$. Also,

$$
\begin{aligned}
d\left(x_{2}, x_{3}\right)=d\left(S x_{1}, T x_{2}\right) & \leq \alpha\left[d\left(x_{2}, S x_{1}\right)+d\left(x_{1}, T x_{2}\right)\right] \\
& =\alpha d\left(x_{1}, x_{3}\right) \\
& \leq \alpha\left[d\left(x_{1}, x_{2}\right)+d\left(x_{2}, x_{3}\right)\right]
\end{aligned}
$$

Therefore, $d\left(x_{2}, x_{3}\right) \leq \frac{\alpha}{1-\alpha} d\left(x_{1}, x_{2}\right) \leq\left(\frac{\alpha}{1-\alpha}\right)^{2} d\left(x_{0}, x_{1}\right)$.

Proceeding similarly we get, $d\left(x_{n}, x_{n+1}\right) \leq\left(\frac{\alpha}{1-\alpha}\right)^{n} d\left(x_{0}, x_{1}\right) \forall n \in \mathbb{N}$. Hence, $d\left(x_{n}, x_{n+1}\right)$ $\rightarrow 0$ as $n \rightarrow \infty$. Hence by Lemma 3 we get both $T$ and $S$ have the approximate fixed point property. 
Theorem 3 Let $(X, d)$ be a metric space and $T, S: X \rightarrow X$ be two self maps such that $d(T x, S y) \leq \alpha_{1} d(x, T x)+\alpha_{2} d(y, S y)+\alpha_{3} d(x, S y)+\alpha_{4} d(y, T x)+\alpha_{5} d(x, y)$ $\forall x, y \in X$, where $\alpha_{i} \geq 0 \forall i=1(1) 5$ with $\alpha=\sum_{i=1}^{5} \alpha_{i}<1$. Then for any $\epsilon>0$, $F_{\epsilon}(T) \neq \emptyset$ and also $F_{\epsilon}(S) \neq \emptyset$; that is both $T$ and $S$ have the approximate fixed point property.

Proof. Let $x_{0} \in X$. We construct the sequence $\left\{x_{n}\right\}$ on $X$ by

$$
x_{n}= \begin{cases}T x_{n-1}, & \text { when } n \text { is odd } \\ S x_{n-1}, & \text { when } n \text { is even }\end{cases}
$$

Now,

$$
\begin{aligned}
& d\left(x_{1}, x_{2}\right) \\
= & d\left(T x_{0}, S x_{1}\right) \\
\leq & \alpha_{1} d\left(x_{0}, T x_{0}\right)+\alpha_{2} d\left(x_{1}, S x_{1}\right)+\alpha_{3} d\left(x_{0}, S x_{1}\right)+\alpha_{4} d\left(x_{1}, T x_{0}\right)+\alpha_{5} d\left(x_{0}, x_{1}\right) \\
\leq & \alpha_{1} d\left(x_{0}, x_{1}\right)+\alpha_{2} d\left(x_{1}, x_{2}\right)+\alpha_{3} d\left(x_{0}, x_{2}\right)+\alpha_{5} d\left(x_{0}, x_{1}\right)
\end{aligned}
$$

Now, $d\left(x_{0}, x_{2}\right) \leq d\left(x_{0}, x_{1}\right)+d\left(x_{1}, x_{2}\right)$ which implies $d\left(x_{0}, x_{2}\right)-d\left(x_{0}, x_{1}\right) \leq d\left(x_{1}, x_{2}\right)$. Now from (2) we get,

$d\left(x_{1}, x_{2}\right) \leq \frac{\alpha_{1}+\alpha_{5}}{1-\alpha_{2}} d\left(x_{0}, x_{1}\right)+\frac{\alpha_{3}}{1-\alpha_{2}} d\left(x_{0}, x_{2}\right)$. Thus we get $d\left(x_{0}, x_{2}\right) \leq \frac{1+\alpha_{1}+\alpha_{5}-\alpha_{2}}{1-\alpha_{2}-\alpha_{3}} d\left(x_{0}, x_{1}\right)$. Hence,

$$
\begin{aligned}
d\left(x_{1}, x_{2}\right) & \leq \frac{\alpha_{1}+\alpha_{5}}{1-\alpha_{2}} d\left(x_{0}, x_{1}\right)+\frac{\alpha_{3}}{1-\alpha_{2}} \frac{1+\alpha_{1}+\alpha_{5}-\alpha_{2}}{1-\alpha_{2}-\alpha_{3}} d\left(x_{0}, x_{1}\right) \\
& =\frac{1}{1-\alpha_{2}}\left[\alpha_{1}+\alpha_{5}+\frac{\alpha_{3}\left(1+\alpha_{1}+\alpha_{5}-\alpha_{2}\right)}{1-\alpha_{2}-\alpha_{3}}\right] d\left(x_{0}, x_{1}\right) \\
& =\frac{\alpha_{1}+\alpha_{3}+\alpha_{5}}{1-\alpha_{2}-\alpha_{3}} d\left(x_{0}, x_{1}\right)
\end{aligned}
$$

Due to symmetry we can change, $\alpha_{1}$ with $\alpha_{2}$ and $\alpha_{3}$ with $\alpha_{4}$. So we get,

$$
d\left(x_{1}, x_{2}\right) \leq \frac{\alpha_{2}+\alpha_{4}+\alpha_{5}}{1-\alpha_{1}-\alpha_{4}} d\left(x_{0}, x_{1}\right)
$$

Let, $\beta=\min \left\{\frac{\alpha_{1}+\alpha_{3}+\alpha_{5}}{1-\alpha_{2}-\alpha_{3}}, \frac{\alpha_{2}+\alpha_{4}+\alpha_{5}}{1-\alpha_{1}-\alpha_{4}}\right\}$. Then from (3) and (4) we get $d\left(x_{1}, x_{2}\right) \leq$ $\beta d\left(x_{0}, x_{1}\right), 0<\beta<1$. In a similar manner we get, $d\left(x_{2}, x_{3}\right) \leq \beta d\left(x_{1}, x_{2}\right) \leq$ $\beta^{2} d\left(x_{0}, x_{1}\right)$. Proceeding in this way we have $d\left(x_{n}, x_{n+1}\right) \leq \beta^{n} d\left(x_{0}, x_{1}\right) \forall n \in \mathbb{N}$. Therefore, $d\left(x_{n}, x_{n+1}\right) \rightarrow 0$ as $n \rightarrow \infty$. Thus by Lemma 3 we see that both $T$ and $S$ have the approximate fixed point property.

Theorem 4 Let $(X, d)$ be a metric space and $T, S: X \rightarrow X$ be two self mappings. Also let $\forall x, y \in X$ atleast one of the following conditions hold

(i) $d(T x, S y) \leq k_{1}[d(x, T x)+d(y, S y)]$;

(ii) $d(T x, S y) \leq k_{2}[d(x, S y)+d(y, T x)]$, where $k_{1}, k_{2} \in\left(0, \frac{1}{2}\right)$.

Then for any $\epsilon>0, F_{\epsilon}(T) \neq \emptyset$ and $F_{\epsilon}(S) \neq \emptyset$; which ensures that both $T$ and $S$ have the approximate fixed point property. 
Proof. Let $x_{0} \in X$. We construct the sequence $\left\{x_{n}\right\}$ on $X$ by

$$
x_{n}= \begin{cases}T x_{n-1}, & \text { when } n \text { is odd } \\ S x_{n-1}, & \text { when } n \text { is even }\end{cases}
$$

Now for $x_{0}, x_{1} \in X$, from (i) we get $d\left(x_{1}, x_{2}\right)=d\left(T x_{0}, S x_{1}\right) \leq k_{1}\left[d\left(x_{0}, T x_{0}\right)+\right.$ $\left.d\left(x_{1}, S x_{1}\right)\right]$, which implies $d\left(x_{1}, x_{2}\right) \leq \frac{k_{1}}{1-k_{1}} d\left(x_{0}, x_{1}\right)$. Again by (ii) $d\left(x_{1}, x_{2}\right)=$ $d\left(T x_{0}, S x_{1}\right) \leq k_{2}\left[d\left(x_{0}, S x_{1}\right)+d\left(x_{1}, T x_{0}\right)\right]$ implying that $d\left(x_{1}, x_{2}\right) \leq \frac{k_{2}}{1-k_{2}} d\left(x_{0}, x_{1}\right)$. Let $k=\max \left\{\frac{k_{1}}{1-k_{1}}, \frac{k_{2}}{1-k_{2}}\right\}$. Then we have, $d\left(x_{1}, x_{2}\right) \leq k d\left(x_{0}, x_{1}\right)$, where $0<k<1$. In a similar manner for $x_{1}, x_{2} \in X$ we get, $d\left(x_{2}, x_{3}\right) \leq k d\left(x_{1}, x_{2}\right) \leq k^{2} d\left(x_{0}, x_{1}\right)$. Proceeding in this way we have $d\left(x_{n}, x_{n+1}\right) \leq k^{n} d\left(x_{0}, x_{1}\right) \forall n \in \mathbb{N}$. Therefore, $d\left(x_{n}, x_{n+1}\right)$ $\rightarrow 0$ as $n \rightarrow \infty$. Therefore by Lemma 3 we get both $T$ and $S$ have the approximate fixed point property.

Theorem 5 Let $(X, d)$ be a metric space and $T, S: X \rightarrow X$ be two mappings, satisfying $d(T x, S y) \leq \beta \max \left\{d(x, y), d(x, T x), d(y, S y), \frac{1}{2}(d(x, S y)+d(y, T x))\right\}$ for all $x, y \in X$, where $0<\beta<1$. Then for any $\epsilon>0, F_{\epsilon}(T) \neq \emptyset$ and also $F_{\epsilon}(S) \neq \emptyset$; equivalently both $T$ and $S$ have the approximate fixed point property.

Proof. Let $x_{0} \in X$ be fixed. Let us construct the sequence $\left\{x_{n}\right\}$ in $X$, defined by $x_{2 n}=S_{2 n-1}$ and $x_{2 n-1}=T x_{2 n-2}$ for all $n \geq 1$. If for some $n \geq 0 x_{2 n}=x_{2 n+1}$, then we have

$$
\begin{aligned}
d\left(x_{2 n+1}, x_{2 n+2}\right)= & d\left(T x_{2 n}, S x_{2 n+1}\right) \\
\leq & \beta \max \left\{d\left(x_{2 n}, x_{2 n+1}\right), d\left(x_{2 n}, T x_{2 n}\right), d\left(x_{2 n+1}, S x_{2 n+1}\right)\right. \\
& \left.\frac{1}{2}\left(d\left(x_{2 n}, S x_{2 n+1}\right)+d\left(x_{2 n+1}\right), T x_{2 n}\right)\right\} \\
= & \beta \max \left\{d\left(x_{2 n+1}, x_{2 n+2}\right), \frac{1}{2} d\left(x_{2 n}, x_{2 n+2}\right)\right\} \\
= & \beta d\left(x_{2 n+1}, x_{2 n+2}\right)
\end{aligned}
$$

So $d\left(x_{2 n+1}, x_{2 n+2}\right)=0$ implying that $x_{2 n+1}=x_{2 n+2}$. Showing that both $T$ and $S$ has a fixed point in $X$. Hence both $T, S$ have the approximate fixed point property. Again if for some $n \in \mathbb{N}, x_{2 n-1}=x_{2 n}$ then we see that both $T, S$ have the approximate fixed point property. Thus without loss of generality, let us suppose that $x_{n} \neq x_{n+1}$ for all $n \geq 0$. Then for all $n \geq 1$,

$$
\begin{aligned}
& d\left(x_{2 n-1}, x_{2 n}\right) \\
= & d\left(T x_{2 n-2}, S x_{2 n-1}\right) \\
\leq & \beta \max \left\{d\left(x_{2 n-2}, x_{2 n-1}\right), d\left(x_{2 n-2}, T x_{2 n-2}\right), d\left(x_{2 n-1}, S x_{2 n-1}\right),\right. \\
& \left.\frac{1}{2}\left(d\left(x_{2 n-2}, S x_{2 n-1}\right)+d\left(x_{2 n-1}, T x_{2 n-2}\right)\right)\right\} \\
= & \beta \max \left\{d\left(x_{2 n-2}, x_{2 n-1}\right), d\left(x_{2 n-1}, x_{2 n}\right), \frac{1}{2} d\left(x_{2 n-2}, x_{2 n}\right)\right\}
\end{aligned}
$$


First suppose that, $\max \left\{d\left(x_{2 n-2}, x_{2 n-1}\right), d\left(x_{2 n-1}, x_{2 n}\right), \frac{1}{2} d\left(x_{2 n-2}, x_{2 n}\right)\right\}=$ $d\left(x_{2 n-2}, x_{2 n-1}\right)$, then from (5) we get $d\left(x_{2 n-1}, x_{2 n}\right) \leq \beta d\left(x_{2 n-2}, x_{2 n-1}\right)$. Again if, $\max \left\{d\left(x_{2 n-2}, x_{2 n-1}\right), d\left(x_{2 n-1}, x_{2 n}\right), \frac{1}{2} d\left(x_{2 n-2}, x_{2 n}\right)\right\}=\frac{1}{2} d\left(x_{2 n-2}, x_{2 n}\right)$, then from (5) we have, $d\left(x_{2 n-1}, x_{2 n}\right) \leq \frac{\beta}{2-\beta} d\left(x_{2 n-2}, x_{2 n-1}\right)<\beta d\left(x_{2 n-2}, x_{2 n-1}\right)$. So in any case $d\left(x_{2 n-1}, x_{2 n}\right) \leq \beta d\left(x_{2 n-2}, x_{2 n-1}\right) \forall n \in \mathbb{N}$. Similarly for any $n \geq 1, d\left(x_{2 n}, x_{2 n+1}\right) \leq$ $\beta d\left(x_{2 n-1}, x_{2 n}\right)$. That is for any $n \in \mathbb{N}$ we have,

$$
\begin{aligned}
d\left(x_{n}, x_{n+1}\right) \leq & \beta d\left(x_{n-1}, x_{n}\right) \\
\leq & \beta^{2} d\left(x_{n-2}, x_{n-1}\right) \\
& \cdots \\
\leq & \beta^{n} d\left(x_{0}, x_{1}\right)
\end{aligned}
$$

So $d\left(x_{n}, x_{n+1}\right) \rightarrow 0$ as $n \rightarrow \infty$. Thus by using Lemma 3 we get both $T$ and $S$ have the approximate fixed point property.

Theorem 6 Let $(X, d)$ be a metric space and $T, S: X \rightarrow X$ be two self mappings satisfying $d(T x, S y) \leq \alpha[d(x, T x)+d(y, S y)] \forall x, y \in X, 0<\alpha<\frac{1}{2}$. If $T$ and $S$ have the c.a.f.p.p. then for any $\epsilon>0, \delta\left(F_{\epsilon}(T, S)\right) \leq 2 \epsilon(1+\alpha)$.

Proof. We know that for any $\epsilon>0, F_{\epsilon}(T) \neq \emptyset$ and $F_{\epsilon}(S) \neq \emptyset$, which follows from Theorem 1.

Now let $\epsilon>0$ be fixed. Also let, for some $\eta>0$ we have $d(x, y)-d(T x, S y) \leq \eta$, for some $x \in F_{\epsilon}(T)$ and $y \in F_{\epsilon}(S)$. Then

$$
\begin{aligned}
d(x, y) & \leq d(T x, S y)+\eta \\
& \leq \alpha[d(x, T x)+d(y, S y)] \\
& <2 \alpha \epsilon+\eta
\end{aligned}
$$

So for all $\eta>0$, there exits a mapping $\phi_{\epsilon}(\eta)=2 \alpha \epsilon+\eta>0$ such that, $d(x, y)-$ $d(T x, S y) \leq \eta \Rightarrow d(x, y) \leq \phi_{\epsilon}(\eta)$, for all $x \in F_{\epsilon}(T)$ and $y \in F_{\epsilon}(S)$. Therefore by Lemma 4 we get, $\delta\left(F_{\epsilon}(T, S)\right) \leq \phi_{\epsilon}(2 \epsilon)=2 \epsilon(1+\alpha)$ for all $\epsilon>0$, if $T$ and $S$ have the c.a.f.p.p. .

Theorem 7 Let $(X, d)$ be a metric space and $T, S: X \rightarrow X$ be two self maps satisfying $d(T x, S y) \leq \alpha[d(x, S y)+d(y, T x)] \forall x \in X, 0<\alpha<\frac{1}{2}$. If $T$ and $S$ have the c.a.f.p.p. then for any $\epsilon>0, \delta\left(F_{\epsilon}(T, S)\right) \leq \frac{2 \epsilon(1+\alpha)}{1-2 \alpha}$.

Proof. It follows from Theorem 2 that both $T$ and $S$ have the approximate fixed point property.

Let $\epsilon>0$ be given. Also let, $\eta>0$ be arbitrary such that, $d(x, y)-d(T x, S y) \leq \eta$, for some $x \in F_{\epsilon}(T)$ and $y \in F_{\epsilon}(S)$. Then,

$$
\begin{aligned}
d(x, y) & \leq d(T x, S y)+\eta \\
\leq & \alpha[d(x, S y)+d(y, T x)]+\eta \\
\leq & \alpha[d(x, y)+d(y, S y)+d(y, x)+d(x, T x)]+\eta
\end{aligned}
$$


which implies that $(1-2 \alpha) d(x, y) \leq \alpha[d(x, T x)+d(x, S y)]+\eta$. Since $x \in F_{\epsilon}(T)$ and $y \in F_{\epsilon}(S)$, we see that $(1-2 \alpha) d(x, y) \leq 2 \alpha \epsilon+\eta$ and therefore $d(x, y) \leq \frac{2 \alpha \epsilon+\eta}{1-2 \alpha}$. Hence for all $\eta>0, \exists \phi_{\epsilon}(\eta)=\frac{2 \alpha \epsilon+\eta}{1-2 \alpha}>0$ such that, $d(x, y)-d(T x, S y) \leq \eta \Rightarrow d(x, y) \leq$ $\phi_{\epsilon}(\eta)$, for all $x \in F_{\epsilon}(T)$ and $y \in F_{\epsilon}(S)$. Thus by Lemma 4 we get, $\delta\left(F_{\epsilon}(T, S)\right) \leq$ $\phi_{\epsilon}(2 \epsilon)=\frac{2 \epsilon(1+\alpha)}{1-2 \alpha}$ for all $\epsilon>0$, provided $T$ and $S$ have the c.a.f.p.p. .

Theorem 8 Let $(X, d)$ be a metric space and $T, S: X \rightarrow X$ be two self mappings such that $d(T x, S y) \leq \alpha_{1} d(x, T x)+\alpha_{2} d(y, S y)+\alpha_{3} d(x, S y)+\alpha_{4} d(y, T x)+\alpha_{5} d(x, y)$ $\forall x, y \in X$, where $\alpha_{i} \geq 0 \forall i=1(1) 5$ with $\alpha=\sum_{i=1}^{5} \alpha_{i}<1$. If T, $S$ have the c.a.f.p.p. then for any $\epsilon>0, \delta\left(F_{\epsilon}(T, S)\right) \leq \frac{\alpha_{1}+\alpha_{2}+\alpha_{3}+\alpha_{4}+2}{1-\alpha_{3}-\alpha_{4}-\alpha_{5}} \epsilon$.

Proof. we see from Theorem 3 that for any $\epsilon>0, F_{\epsilon}(T) \neq \emptyset$ and $F_{\epsilon}(S) \neq \emptyset$.

Let $\epsilon>0$ be given. Also let for some $\eta>0$, we have $d(x, y)-d(T x, S y) \leq \eta$, for some $x \in F_{\epsilon}(T)$ and $y \in F_{\epsilon}(S)$. Now,

$$
\begin{aligned}
& d(T x, S y) \\
\leq & \alpha_{1} d(x, T x)+\alpha_{2} d(y, S y)+\alpha_{3} d(x, S y)+\alpha_{4} d(y, T x)+\alpha_{5} d(x, y) \\
\leq & \alpha_{1} d(x, T x)+\alpha_{2} d(y, S y)+\alpha_{3} d(x, T x)+\alpha_{3} d(T x, S y)+\alpha_{4} d(y, S y)+ \\
& \alpha_{4} d(S y, T x)+\alpha_{5} d(x, y) \\
\leq & \frac{\alpha_{1}+\alpha_{3}}{1-\alpha_{3}-\alpha_{4}} d(x, T x)+\frac{\alpha_{2}+\alpha_{4}}{1-\alpha_{3}-\alpha_{4}} d(y, S y)+\frac{\alpha_{5}}{1-\alpha_{3}-\alpha_{4}} d(x, y)
\end{aligned}
$$

Then using (6) we get, $d(x, y) \leq d(T x, S y)+\eta \leq \frac{\alpha_{1}+\alpha_{3}}{1-\alpha_{3}-\alpha_{4}} d(x, T x)+\frac{\alpha_{2}+\alpha_{4}}{1-\alpha_{3}-\alpha_{4}} d(y, S y)+$ $\frac{\alpha_{5}}{1-\alpha_{3}-\alpha_{4}} d(x, y)+\eta$, which implies that, $d(x, y) \leq \frac{\alpha_{1}+\alpha_{2}+\alpha_{3}+\alpha_{4}}{1-\alpha_{3}-\alpha_{4}-\alpha_{5}} \epsilon+\frac{1}{1-\alpha_{3}-\alpha_{4}-\alpha_{5}} \eta$. So for all $\eta>0$ there exists $\phi_{\epsilon}(\eta)=\frac{\alpha_{1}+\alpha_{2}+\alpha_{3}+\alpha_{4}}{1-\alpha_{3}-\alpha_{4}-\alpha_{5}} \epsilon+\frac{1}{1-\alpha_{3}-\alpha_{4}-\alpha_{5}} \eta>0$ such that, $d(x, y)-d(T x, S y) \leq \eta \Rightarrow d(x, y) \leq \phi_{\epsilon}(\eta)$, for all $x \in F_{\epsilon}(T)$ and $y \in F_{\epsilon}(S)$. So by using Lemma 4 we get, $\delta\left(F_{\epsilon}(T, S)\right) \leq \phi_{\epsilon}(2 \epsilon)=\frac{\alpha_{1}+\alpha_{2}+\alpha_{3}+\alpha_{4}+2}{1-\alpha_{3}-\alpha_{4}-\alpha_{5}} \epsilon$ for all $\epsilon>0$, if $T$ and $S$ have the c.a.f.p.p. .

Theorem 9 Let $(X, d)$ be a metric space and $T, S: X \rightarrow X$ be two self mappings. Also let us assume that $\forall x, y \in X$ atleast one of the following conditions hold:

(i) $d(T x, S y) \leq k_{1}[d(x, T x)+d(y, S y)]$;

(ii) $d(T x, S y) \leq k_{2}[d(x, S y)+d(y, T x)]$, where $k_{1}, k_{2} \in\left(0, \frac{1}{2}\right)$.

Then for any $\epsilon>0, \delta\left(F_{\epsilon}(T, S)\right) \leq 2 \frac{1+k}{1-k} \epsilon$, where $k=\max \left\{\frac{k_{1}}{1-k_{1}}, \frac{k_{2}}{1-k_{2}}\right\}$, if $T$ and $S$ have the c.a.f.p.p. .

Proof. It follows from Theorem 4 that for any $\epsilon>0, F_{\epsilon}(T) \neq \emptyset$ and $F_{\epsilon}(S) \neq \emptyset$. Let, $x, y \in X$.

Case I. Let (i) holds then,

$$
\begin{aligned}
d(T x, S y) & \leq k_{1}[d(x, T x)+d(y, S y)] \\
& \leq k_{1} d(x, T x)+k_{1}[d(y, x)+d(x, T x)+d(T x, S y)] \\
& \leq \frac{2 k_{1}}{1-k_{1}} d(x, T x)+\frac{k_{1}}{1-k_{1}} d(x, y)
\end{aligned}
$$


Also,

$$
\begin{aligned}
d(T x, S y) & \leq k_{1}[d(x, T x)+d(y, S y)] \\
& \leq k_{1}[d(x, y)+d(y, S y)+d(T x, S y)]+k_{1} d(y, S y) \\
& \leq \frac{2 k_{1}}{1-k_{1}} d(y, S y)+\frac{k_{1}}{1-k_{1}} d(x, y)
\end{aligned}
$$

Case II. Let (ii) holds then,

$$
\begin{aligned}
d(T x, S y) & \leq k_{2}[d(x, S y)+d(y, T x)] \\
& \leq k_{2}[d(x, y)+d(y, S y)]+k_{2}[d(y, S y)+d(S y, T x)] \\
& \leq \frac{2 k_{2}}{1-k_{2}} d(y, S y)+\frac{k_{2}}{1-k_{2}} d(x, y)
\end{aligned}
$$

and also,

$$
\begin{aligned}
d(T x, S y) & \leq k_{2}[d(x, S y)+d(y, T x)] \\
& \leq k_{2}[d(x, T x)+d(T x, S y)]+k_{2}[d(y, x)+d(x, T x)] \\
& \leq \frac{2 k_{2}}{1-k_{2}} d(x, T x)+\frac{k_{2}}{1-k_{2}} d(x, y)
\end{aligned}
$$

Let $k=\max \left\{\frac{k_{1}}{1-k_{1}}, \frac{k_{2}}{1-k_{2}}\right\}$. If either (i) or (ii) holds then $\forall x, y \in X$ we get,

$$
\begin{aligned}
& d(T x, S y) \leq 2 k d(x, T x)+k d(x, y) ; \\
& d(T x, S y) \leq 2 k d(y, S y)+k d(x, y) .
\end{aligned}
$$

Let $\epsilon>0$ be given. Also assume that for some $\eta>0, d(x, y)-d(T x, S y) \leq \eta$, for some $x \in F_{\epsilon}(T)$ and $y \in F_{\epsilon}(S)$. Then using (7) we get, $d(x, y) \leq \frac{2 k \epsilon+\eta}{1-k}$. Now setting $\phi_{\epsilon}(\eta)=\frac{2 k \epsilon+\eta}{1-k}$ for all $\eta>0$, we see that $d(x, y)-d(T x, S y) \leq \eta \Rightarrow d(x, y) \leq \phi_{\epsilon}(\eta)$, for all $x \in F_{\epsilon}(T)$ and $y \in F_{\epsilon}(S)$. Therefore by Lemma 4 we get, $\delta\left(F_{\epsilon}(T, S)\right) \leq$ $\phi_{\epsilon}(2 \epsilon)=2 \frac{1+k}{1-k} \epsilon$, for all $\epsilon>0$, provided $T$ and $S$ have the c.a.f.p.p. .

Theorem 10 Let $(X, d)$ be a metric space and $T, S: X \rightarrow X$ be two mappings such that $d(T x, S y) \leq \beta \max \left\{d(x, y), d(x, T x), d(y, S y), \frac{1}{2}(d(x, S y)+d(y, T x))\right\}$ for all $x, y \in X$, where $0<\beta<1$. If $T$ and $S$ have the c.a.f.p.p. then for any $\epsilon>0$, $\delta\left(F_{\epsilon}(T, S)\right) \leq \max \left\{\frac{2 \epsilon}{1-\beta},(2+\beta) \epsilon, \frac{2-\beta}{1-\beta} \epsilon\right\}$.

Proof. Theorem 5 ensures that both $T$ and $S$ have the approximate fixed point property. Let $\epsilon>0$ be fixed. Also assume that for some $\eta>0$ and for $x \in F_{\epsilon}(T)$, $y \in F_{\epsilon}(S) T, S$ satisfy $d(x, y)-d(T x, S y) \leq \eta$. Now,

$$
\begin{aligned}
d(T x, S y) & \leq \beta \max \left\{d(x, y), d(x, T x), d(y, S y), \frac{1}{2}(d(x, S y)+d(y, T x))\right\} \\
& <\beta \max \left\{d(x, y), \epsilon, \epsilon, \frac{1}{2}(2 \epsilon+2 d(T x, S y))\right\} \\
& =\beta \max \{d(x, y), \epsilon, \epsilon+d(T x, S y)\}
\end{aligned}
$$


If, $\max \{d(x, y), \epsilon, \epsilon+d(T x, S y)\}=d(x, y)$ then $d(T x, S y)<\beta d(x, y)$, implying that $d(x, y)<\frac{\eta}{1-\beta}$. For the next two respective cases we have $d(x, y)<\beta \epsilon+\eta$ and $d(x, y)<\frac{\beta}{1-\beta} \epsilon+\eta$. Therefore $d(x, y) \leq \phi_{\epsilon}(\eta)$, where $\phi_{\epsilon}(\eta)=\max \left\{\frac{\eta}{1-\beta}, \beta \epsilon+\right.$ $\left.\eta, \frac{\beta}{1-\beta} \epsilon+\eta\right\}$. Then by Lemma 4 we get, if $T$ and $S$ have the c.a.f.p.p. then for any $\epsilon>0, \delta\left(F_{\epsilon}(T, S)\right) \leq \phi_{\epsilon}(2 \epsilon)=\max \left\{\frac{2 \epsilon}{1-\beta},(2+\beta) \epsilon, \frac{2-\beta}{1-\beta} \epsilon\right\}$.

Remark 3 Actually Theorem 1 and Theorem 2 are special cases of Theorem 3. Similarly Theorem 6 and Theorem 7 are special cases of Theorem 8. If we put $\alpha_{3}=\alpha_{4}=\alpha_{5}=0$ in Theorem 3 and in Theorem 8 we get the corresponding results of Theorem 1 and Theorem 6. In a similar manner if we put $\alpha_{1}=\alpha_{2}=\alpha_{5}=0$ in Theorem 3 and in Theorem 8 we get the results of Theorem 2 and Theorem 7 respectively.

Corollary 1 Suppose that in a metric space $(X, d)$ two mappings $T$ and $S$ satisfy the condition of Theorem 3 (or, Theorem 8) and posses a common fixed point $x^{*}$. Then

(i) $x^{*}$ is the unique common fixed point of $T$ and $S$.

(ii) for each sequence $\left\{x_{n}\right\}$ with the property that for each $n \in \mathbb{N}$, the point $x_{n}$ is a common $\frac{1}{n}$-fixed point of $T$ and $S$, we have $\lim x_{n}=x^{*}$.

Proof. (i) follows trivially.

(ii) Since $x^{*}$ is a common fixed point of $T$ and $S$ then it is clear that for any $\epsilon>0$ $x^{*}$ is a common $\epsilon$-fixed point. Therefore $T$ and $S$ have the c.a.f.p.p. and so for all $\epsilon>0, \delta\left(F_{\epsilon}(T, S)\right) \leq \frac{\alpha_{1}+\alpha_{2}+\alpha_{3}+\alpha_{4}+2}{1-\alpha_{3}-\alpha_{4}-\alpha_{5}} \epsilon$, follows from Theorem 3 (or, Theorem 8). Now it is given that the point $x_{n}$ is a common $\frac{1}{n}$-fixed point for each $n \in \mathbb{N}$, then we see that, $d\left(x_{n}, x^{*}\right) \leq \delta\left(F_{\epsilon}(T, S)\right) \leq\left(\frac{\alpha_{1}+\alpha_{2}+\alpha_{3}+\alpha_{4}+2}{1-\alpha_{3}-\alpha_{4}-\alpha_{5}}\right) \frac{1}{n}$. Hence $\lim x_{n}=x^{*}$.

Remark 4 In a complete metric space if two mappings $T$ and $S$ satisfy any of the contractive conditions given in Theorem 1, Theorem 2, Theorem 3 and Theorem 4 , then they have a unique common fixed point in $X$. Therefore clearly they have c.a.f.p.p. and thus Theorem 6 to Theorem 9 also hold good.

Example 1 Let $X=(0, \infty)$ be the metric space with usual metric. Let us take two mappings $T, S: X \rightarrow X$ given by $T x=\frac{x}{10}$ and $S x=\frac{x}{20}$ for all $x \in X$. Then they satisfies the condition of Theorem 1 for the constant $\alpha=\frac{1}{9}$. For any $\epsilon>0$, we get $F_{\epsilon}(T)=\left(0, \frac{10}{9} \epsilon\right)$ and $F_{\epsilon}(S)=\left(0, \frac{20}{19} \epsilon\right)$. Also, $F_{\epsilon}(T, S)=\left(0, \frac{20}{19} \epsilon\right)$, therefore the mappings have the c.a.f.p.p. . Note that they have no common fixed point in $X$.

Example 2 Let $X=(0,1]$ be the metric space equipped with usual metric of reals. Let $T, S: X \rightarrow X$ be mappings defined by

$$
T(x)=\left\{\begin{array}{ll}
\frac{x}{4}, & x \neq 1 \\
\frac{1}{5}, & x=1
\end{array}, S(x)= \begin{cases}\frac{x}{5}, & x \neq 1 \\
\frac{1}{4}, & x=1\end{cases}\right.
$$


Then $T$ and $S$ satisfy the condition of Theorem 1 with the Lipschitz constant $\alpha=\frac{1}{3}$. For any $0<\epsilon \leq \frac{4}{5}, F_{\epsilon}(T)=\left(0, \frac{4}{3} \epsilon\right) \cap(0,1)$ and for any $\epsilon>\frac{4}{5}$ we have $F_{\epsilon}(T)=$ $\left[\left(0, \frac{4}{3} \epsilon\right) \cap(0,1)\right] \cup\{1\}$. Also if $0<\epsilon \leq \frac{3}{4}$ then $F_{\epsilon}(S)=\left(0, \frac{5}{4} \epsilon\right)$ and whenever $\epsilon>\frac{3}{4}$ then $F_{\epsilon}(S)=\left[\left(0, \frac{5}{4} \epsilon\right) \cap(0,1)\right] \cup\{1\}$. So

$$
F_{\epsilon}(T, S)= \begin{cases}\left(0, \frac{5}{4} \epsilon\right), & \text { if } 0<\epsilon \leq \frac{3}{4} \\ \left(0, \frac{5}{4} \epsilon\right) \cap(0,1), & \text { if } \frac{3}{4}<\epsilon \leq \frac{4}{5} \\ (0,1], & \text { if } \epsilon>\frac{4}{5}\end{cases}
$$

Hence $T$ and $S$ have the c.a.f.p.p. but they have no common fixed point in $X$.

\section{Conclusions}

In this paper the existence of approximate fixed points for pair of self mappings satisfying several contractive conditions over a metric space without being complete has been examined. Also some results on common approximate fixed points for different pair of mappings with contractivity conditions have been proved by showing the existence of two nonempty sets consisting of approximate fixed points for such pair of mappings. It is also worth mentioning that a common fixed point can be obtained as limit of a particular sequence of common approximate fixed points of a pair of mappings satisfying some contractive condition via the diminishing diameter property of set of all common approximate fixed points.

\section{Acknowledgments}

The first author wishes to thank the Council of Scientific and Industrial Research, New Delhi, India, for providing the research fellowship to continue the research work. Also authors are thankful to Dr. Debashis Dey for helping them in preparing this manuscript. Also authors are very much thankful to the learned referees for their valuable comments and suggestions in the improvement of this manuscript.

\section{References}

[1] M. Berinde, Approximate Fixed Point Theorems, Stud. Univ. "Babes-Bolayi" Mathematica, vol. 1, 2006, 11-25.

[2] D. Dey, M. Saha, Approximate Fixed Point of Reich Operator, Acta Math. Univ. Comenianae, vol. 1, 2013, 119-123.

[3] D. Dey, A. K. Laha, M. Saha, Approximate coincidence point of two non-linear mappings, Journal of Mathematics, Hindawi Publishing Corporation, Article ID 962058, 2013. 
[4] W. S. Du, F. Khojasteh, New results and generalizations for approximate fixed point property and their applications, Abstract and Applied Analysis, Article ID 581267, 2014.

[5] W. S. Du, On approximate coincidence point properties and their applications to fixed point theory, Journal of Applied Mathematics, Article ID 302830, 2012.

[6] M. A. Miandaragh, M. Postolache, S. Rezapour, Some approximate fixed point results for generalized $\alpha$-contractive mappings, U.P.B. Sci. Bull., Series A, vol. 75 , no. $2,2013$.

[7] B. E. Rhoades, A Comparison of Various Definitions of Contractive Mappings, Trans. Amer. Math. Soc., vol. 226, 1977, 257-290.

[8] S. Tijs, A. Torre, R. Branzei, Approximate Fixed Point Theorems, Libertas Mathematica, vol. 23, 2003, 35-39.

\section{Kushal Roy}

The University of Burdwan

Department of Mathematics

Purba Bardhaman-713104, West Bengal, India

e-mail: kushal.roy93@gmail.com

\section{Mantu Saha}

The University of Burdwan

Department of Mathematics

Purba Bardhaman-713104, West Bengal, India

e-mail: mantusaha.bu@gmail.com 